\title{
Fibre Diffraction Analysis of Skin Offers a Very Early and Extremely Accurate Diagnostic Test for Prostate Cancer
}

\author{
Veronica J. James ${ }^{1}$ and Judith M. O’Malley Ford ${ }^{2}$ \\ ${ }^{1}$ Research School of Chemistry, Australian National University, Canberra, ACT 0200, Australia \\ ${ }^{2}$ Kedron 7 Day Medical Centre, Kedron, Brisbane, QLD 4005, Australia \\ Correspondence should be addressed to Veronica J. James; veronica.james@anu.edu.au
}

Received 30 May 2014; Revised 27 August 2014; Accepted 3 September 2014; Published 17 September 2014

Academic Editor: Mario Budroni

Copyright (c) 2014 V. J. James and J. M. O’Malley Ford. This is an open access article distributed under the Creative Commons Attribution License, which permits unrestricted use, distribution, and reproduction in any medium, provided the original work is properly cited.

\begin{abstract}
Double blind analysis of a batch of thirty skin tissue samples from potential prostate cancer sufferers correctly identified all "control" patients, patients with high and low grade prostate cancers, the presence of benign prostate hyperplasia (BPH), perineural invasions, and the one lymphatic invasion. Identification was by analysis of fibre diffraction patterns interpreted using a schema developed from observations in nine previous studies. The method, schema, and specific experiment results are reported in this paper, with some implications then drawn.
\end{abstract}

\section{Introduction}

This paper is in four main parts:

(1) discussion of the application of fibre diffraction analysis to tissue samples;

(2) summary of results of previous studies and presentation of a diagnostic schema based upon them;

(3) method and results for the latest batch of 30 samples;

(4) discussion and implications.

\section{Fibre Diffraction Analysis}

Fibre diffraction analysis (FDA) relies on patterns produced by the scattering of electrons by X-rays passing through materials that have some regular crystalline structure. It is commonly used in determining the structure of crystals, minerals, flaws in metals, and the like but it can also be applied to animal connective tissues such as hair and muscle. FDA of collagen in the nineteen thirties discovered that when stretching a collagen Type 1 fibril from a rat-tail tendon, the structure becomes pseudo crystalline in three dimensions. Unlike a tendon, "skin collagen" is arranged in a two-dimensional lattice in the dermal layer of skin [1]. If stretched, skin becomes approximately aligned enabling FDA.

Pattern images produced by FDA typically have these features:

(a) a central image of the sample, with its holder often evident as a dark radial line;

(b) a series of surrounding "spreading ripples" from the interference effects produced when the radiations pass through the pseudocrystalline structure;

(c) for closer ripples, a series of full circles;

(d) for more distant ripples, a predominance of ring segments or arcs along one axis with perhaps some patterning along the orthogonal axis and little between.

Such ripple effects are an interference pattern formed by radiation passing through well-ordered gap-overlaps along the collagen fibrils. The gap-overlap for skin was determined as $62.6 \mathrm{~nm}[2,3]$ in 1980. 
An image for a sample of normal skin is shown in Figure 1. Moving outwards from the sample at the dark centre we see the following:

(a) Several arcs that are more evident, particularly the labelled twelfth, fifteenth, and twentieth rings.

(b) Full rings then arcs crossing the meridional (vertical) axis. The angular lengths of arcs are related to the degree of alignment achieved in sample stretching.

(c) The zones between the 12th and the 20th rings on either side of the meridional axis are of most interest. It is here that distinctive effects are seen from irradiation of normal skin samples from those with prostate cancer. a flaring artefact and some full rings initially but these become indistinct along the horizontal (equatorial) axis as well as in regions above and below this axis.

(d) The equatorial pattern, a set of spot-like reflections, becomes slightly distorted or is mostly smeared into broad arcs because the sample is stretched in one direction only.

(e) The position of these reflections relates to the diameters of the collagen helices and their distances apart.

(f) Changes in this normal skin pattern have been observed in patients with a large number of diseases including ageing, insulin dependent diabetes (IDDM), breast cancer, osteogenesis imperfecta, melanoma and nonmelanoma skin cancers, and radiation hypersensitivity [4-11]. Ageing, IDDM, osteogenesis imperfecta, nonmelanoma skin cancers, and radiation hypersensitivity alter the intensities of the reflections in the meridional or equatorial collagen pattern but do not produce complete rings superimposing on the normal pattern.

(g) Full "rings of change" have only been associated with the presence of cancers at this time. Different diameters of rings visible at different diameters are specific to different cancers. Such rings are found in the lateral regions and can be located accurately by their positions relative to the order of the closest meridional arcs. Such "rings of change" appear at the beginning of the cancer, modify with the progression of the cancer, and disappear if and when the cancer has been completely removed [12-14].

\section{Summary of Results of Earlier Studies}

The first "prostate cancer" ring appeared in a study designed for the diagnosis of melanoma [11]. In one of the FDA studies the results of two patients showed not only the "melanoma" ring, (a sharp ring on the sixteenth meridional order), but also a second ring between the 13th and the 14th meridional orders. After completion of the analysis and checking the pathology for these two patients, it was discovered that these two male patients in their middle sixties not only had repeat melanomas but also had low grade prostate cancer. If skin

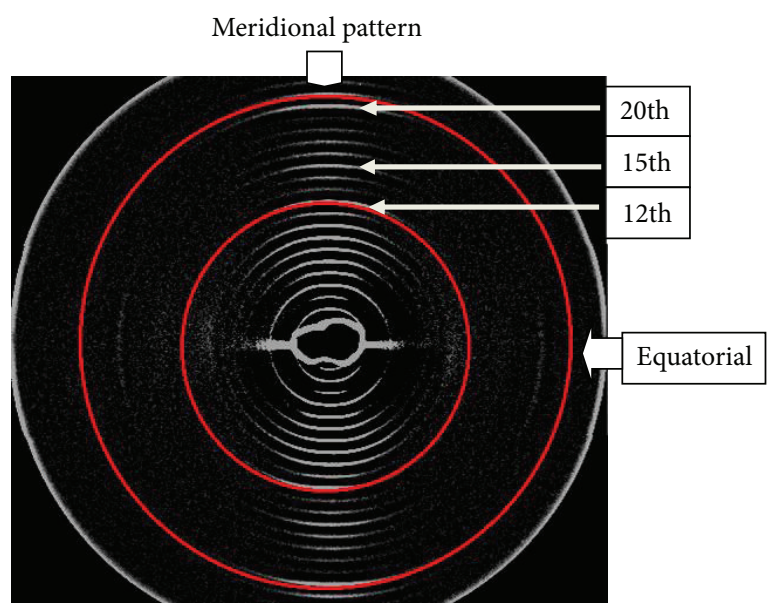

FIGURE 1: Diffraction pattern for normal skin. The region of interest for prostate cancer is the donut between the 12th and the 20th meridional reflections.

cancers are excluded, prostate cancer is the most commonly occurring cancer in men over 50 years of age in Australia [15] resulting in many deaths and it is second only to lung cancer worldwide [16]. It was imperative to follow up on these results.

In our first blinded test using 229 samples, all 13 positive prostate cancer samples were identified suggesting that FDA might be used as a possible diagnostic test [10]. Our next three tests, 116 samples, 67 controls, showed a possible difference between high and low grade prostate cancers and a possible BPH change. Two of these tests also verified the disappearance of the prostate changes when cancers were cured by surgical removal or by chemotherapy and radiation [14]. Extra changes were noted as possibly correlating with perineural or lymphatic invasions. Relying on the origins for diagnosis of these changes, two false positives were noted.

The changes from the normal skin pattern in the presence of cancer sighted in the earlier studies are rings superimposed on the normal pattern. Since the intensity pattern of the meridional arcs is unaltered, it is concluded that the extra molecule that gives rise to these rings do not bind to the helical sections of the skin collagen.

A further blinded study of transgenic adenocarcinoma of the mouse prostate (TRAMP) mice (32 controls, 32 affected) showed that the change in the diffraction pattern is visible at 3 weeks of age, long before any other test can detect prostate cancer [13], so, as in the case of breast cancer [1, 17], "the false positives" may prove to be prostate cancer with longer clinical followup. This mouse test also verified the difference observed, in the previous human studies, between high and low grade prostate cancers.

3.1. Diagnostic Schema. The possible origins of all the changes found in these earlier studies based on the correlation of histology results and changes in the earlier diffraction patterns have been identified and are listed in Table 1. Based on this schema, diagnosis of 30 samples was undertaken using the FDA image for each, as is discussed next. 
TABLE 1

\begin{tabular}{lcc}
\hline CHANGE (in ring pattern) & Expected ORIGIN & Illustration \\
\hline Pattern for controls & Normal pattern & Figure 2 \\
A ring superimposed between the 13th and 14th meridional orders & Low grade prostate cancer & Figure 3 [11] \\
A ring superimposed between the 15th and 16th meridional orders & BPH (benign prostate hypertrophy) & Figure 3 [14] \\
A wide ring centered between the 14th and 15th meridional orders & High grade prostate cancer & Figure 4 unpublished \\
A sharp ring on the 17th meridional order & Perineural invasion & Figure 5 unpub. \\
A sharp ring on the 18th meridional order & Lymphatic invasion & Figure 5 unpub. \\
\hline
\end{tabular}

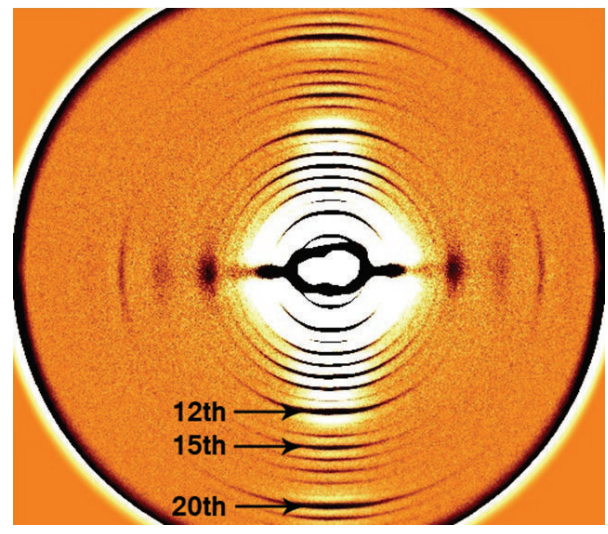

Figure 2: Normal skin diffraction pattern found in Sample 25 in Table 2. There are no rings in this pattern in the region of interest.

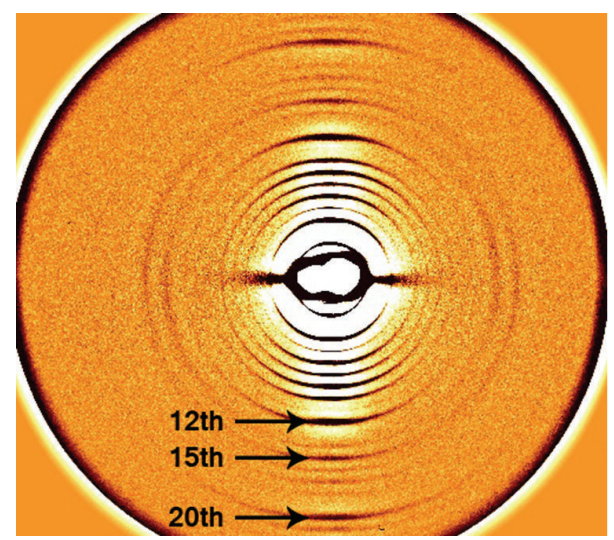

FIGURE 3: If low angle prostate cancer is present in the patient, a ring appears in the FDA pattern situated between the thirteenth and the fourteenth meridional arcs.. This patient also had an enlarged prostate $(\mathrm{BPH})$ which results in the second ring which is situated between the 15th and the 16th meridional orders.

\section{Method and Summary of Results}

4.1. Method. The patient's consent form was completed by each of the 30 consecutive patients undergoing either prostate biopsy or radical prostatectomies who gave skin samples at Wesley Hospital where ethical approval had been granted by the Ethics Board.

These $3 \mathrm{~mm}$ skin punch biopsies were taken using a Keyes punch from the patients at locations on stomach or buttocks

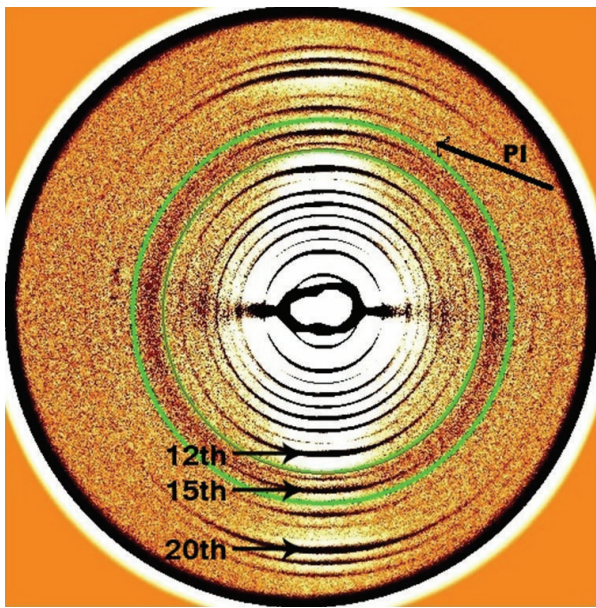

FIGURE 4: For high grade cancer patients, the ring between the 13th and the 14th orders becomes much wider, centring on the 14th order and then beyond this to the 15 th order. The perineural invasion ring is also visible in this figure.

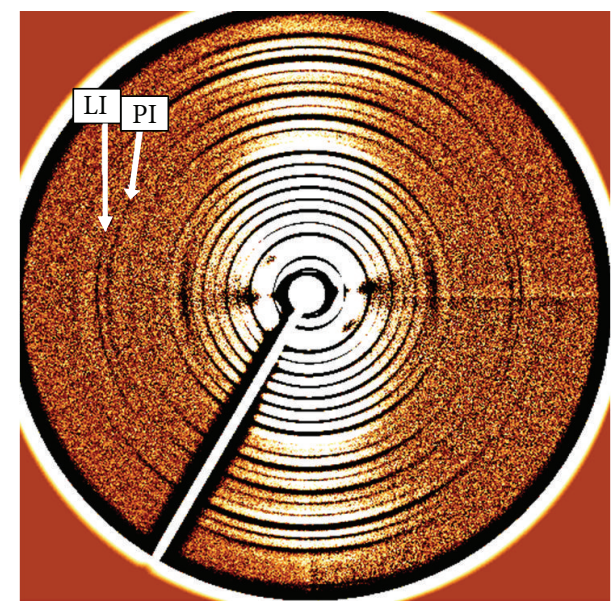

FIGURE 5: Rings for both perineural and lymphatic invasions are present and indicated by PI and LI, respectively.

away from the prostate itself. Samples were immediately placed in small glass bottles containing small quantities of phosphate-buffered saline and identified by numbers only. This simple minimally invasive procedure was done with the assistance of local anaesthetic injection to the area and caused no side effects for these patients. 
TABLE 2

\begin{tabular}{|c|c|c|}
\hline Sample & Diffraction diagnosis & Pathology results \\
\hline 1 & $\mathrm{HGPC}+\mathrm{BPH}+$ perineural invasion & Gleason 7 + invasion (perineural) \\
\hline 2 & $\mathrm{HGPC}+\mathrm{BPH}+$ perineural invasion & BPH, Gleason 7 + invasion (perineural) \\
\hline 3 & HGPC + weak perineural invasion & Gleason $7+$ invasion (perineural) \\
\hline 4 & HGPC + weak perineural invasion & Gleason 7 + invasion (perineural) \\
\hline 5 & HGPC + perineural invasion & Gleason $7+$ invasion (perineural), PSA = 16 \\
\hline 6 & HGPC + perineural and lymphatic invasions & Gleason $7+$ invasion (perineural and lymphatic) PSA $=4.7$ \\
\hline 7 & Gleason $6+$ perineural invasion & Gleason $6+$ invasion (perineural), PSA = 11 \\
\hline 8 & HGPC + perineural invasion & Gleason $9+$ invasion (perineural) \\
\hline 9 & HGPC + no invasions & Gleason $9+$ no invasions \\
\hline 10 & HGPC + no invasions & Gleason 7 + no invasions \\
\hline 11 & HGPC + perineural invasion & Gleason $8+$ invasion (perineural), PSA $=1.8$ \\
\hline 12 & HGPC + perineural invasion & Gleason $7+$ invasion (perineural) \\
\hline 13 & HGPC + perineural invasion & Gleason $6+$ invasion (perineural), PSA $=2.6$ \\
\hline 14 & HGPC + perineural invasion & Gleason 7 + invasion (perineural) \\
\hline 15 & $\mathrm{HGPC}+$ perineural invasion & Gleason 6 + invasion (perineural) \\
\hline 16 & HGPC + no invasions & Prostatic adenocarcinoma + no invasions identified \\
\hline 17 & Weak Gleeson 6 + no invasions & Gleason $6+$ no invasions, PSA $=5.9$ \\
\hline 18 & Weak Gleason 6 + perineural invasion & Gleason $6+$ invasion (perineural), PSA $=0.84$ \\
\hline 19 & HGPC + no invasion & Gleason $7+$ no invasions \\
\hline 20 & Gleason $6+$ no invasion & Gleason $6+$ no invasions, PSA $=5.9$ \\
\hline 21 & HGPC + perineural invasion & Gleason 7 + invasion (perineural) \\
\hline 22 & HGPC + perineural invasion & Gleason 7 + invasion (perineural) \\
\hline 23 & HGPC, perineural invasion & HGPC Gleason $7+$ invasion (perineural), PSA $=6.9$ \\
\hline 24 & HGPC + perineural invasion & Gleason $7+$ invasion (perineural), PSA = 4.9 \\
\hline 25 & Normal & Normal, PSA = 3.2 \\
\hline 26 & $\mathrm{BPH}$, otherwise normal & Benign prostatic tissue \\
\hline 27 & $\mathrm{PC}$ and $\mathrm{BPH}+$ no invasion rings & Prostatic intraepithelial neoplasia no invasions \\
\hline 28 & Normal & Normal, PSA = 6 \\
\hline 29 & Normal & Benign prostatic tissue, $\mathrm{PSA}=4.9$ \\
\hline 30 & HGPC, perineural invasion & HGPC Gleason $8+$ invasion (perineural), PSA $=48.0$ \\
\hline
\end{tabular}

The numbered samples were immediately placed in a freezer and stored at $-20^{\circ}$ Celsius until required at the synchrotron. To achieve double blinding, these frozen samples were then supplied to the diffraction team by a nurse in the Wesley Hospital. No clinical information about the samples was supplied with them. All available details were only supplied when unblinding occurred at the end of the scientific investigation.

The samples were placed in cells specially designed to maintain $100 \%$ humidity throughout the exposure and to enable the sample to be stretched to remove the natural crimp [1]. Diffraction patterns were obtained at the small angle X-ray scattering beamline of the BioCAT facility, Advanced Photon Source, Argonne National Laboratory, USA. Samples were exposed to the beam for 5 seconds at each of the 20 positions along the sample length. The best patterns so obtained were kept. Background was removed using IRAF and SAO, two astronomy programs developed by the Smithsonian Institute for locating stars over a very wide intensity distribution. These programs are ideal for removing the background from the extremely wide intensity distribution of the fiber diffraction pattern of skin collagen.
4.2. Results. The results from the 30 samples in the latest study based on the diagnostic schema summarised in Table 1 are given in Table 2 .

A summary of the results obtained from this final study of 30 samples is as follows.

(1) All 20 perineural and the one lymphatic invasion were correctly identified.

(2) All normal and BPH samples were correctly identified.

(3) All high and low grade prostate cancers were correctly identified.

(4) Sample 27, however, showed the pattern in Figure 3, which was a very strong low grade prostate cancer and BPH.

Histology showed this sample as prostatic intraepithelial neoplasia, which is a diagnosis stating that this patient will have prostate cancer in the future but does not have it now. Therefore this result may not be a false positive but just an earlier diagnosis. Time will tell [17]. 

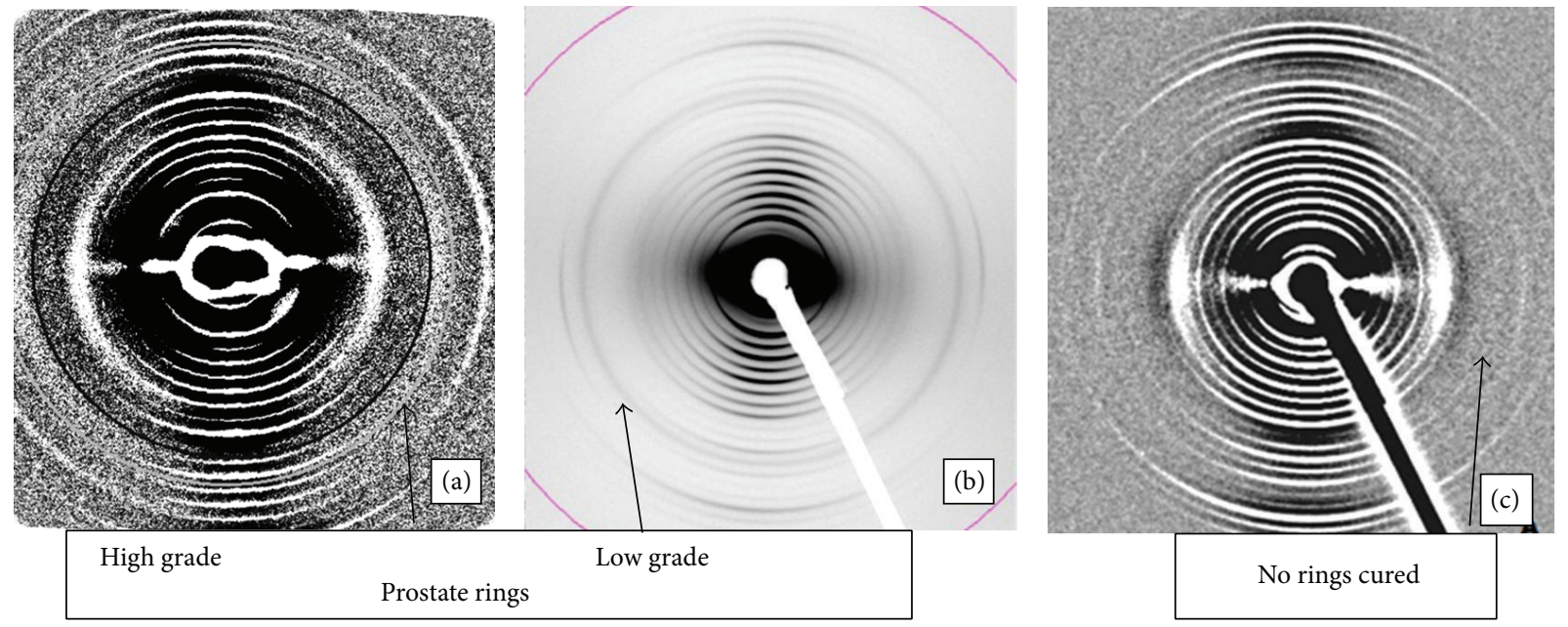

Figure 6

\section{Discussion}

The results from this followup study of 30 samples confirm the accuracy of this diagnostic schema. No PSA and no prostate biopsies were needed. When the results of this test are added to the 347 tests already to hand, the combined sensitivity is $100 \%$ as defined by $\{[\mathrm{TP} /(\mathrm{TP}+\mathrm{FN})] * 100\}$, where $\mathrm{TP}=$ number of true positives and $\mathrm{FN}=$ number of false negatives. However as there were 3 false positives, the specificity is $99.2 \%$, as defined by $\{[\mathrm{TN} /(\mathrm{FP}+\mathrm{TN})] * 100\}$, where $\mathrm{TN}=$ number of true negatives and $\mathrm{FP}=$ number of false positives.

Since a blinded study of transgenic adenocarcinoma of the mouse prostate (TRAMP) mice showed that the change in the diffraction pattern is visible long before any other test can detect prostate cancer [13], "the false positives" may prove to be prostate cancer with a longer clinical followup. During our research we have found that most patients present too late for treatment which enables a cure. For example, only ten of the thirty patients in this test did not have prostate cancers that had invaded beyond the prostate.

This late presentation appears to be responsible for the high death rate from this cancer in Australia. This could be avoided if diagnostic FDA tests were available earlier. With an annual or biennial check, any prostate cancers could be picked up and removed before they have moved outside the prostate itself. This skin test may also determine when a cure has been achieved, as the prostate rings can no longer be seen following treatment if the cancer is removed entirely [14].

In our earlier studies, we had come across 5 patients who have been cured by treatments. One of these five patients, who had undergone 13 prostate biopsies which were all positive and ten of which gave Gleason scores between 7 and 9, did not accept the option of a prostatectomy for fear of possible invasions. He accepted chemotherapy and radiation [13] and was cured according to a later FDA test. Another of these 5 patients, who had a small prostate cancer distant from the surface of the prostate, had a full prostatectomy after advice from his urologist. Both men were cured by their treatments

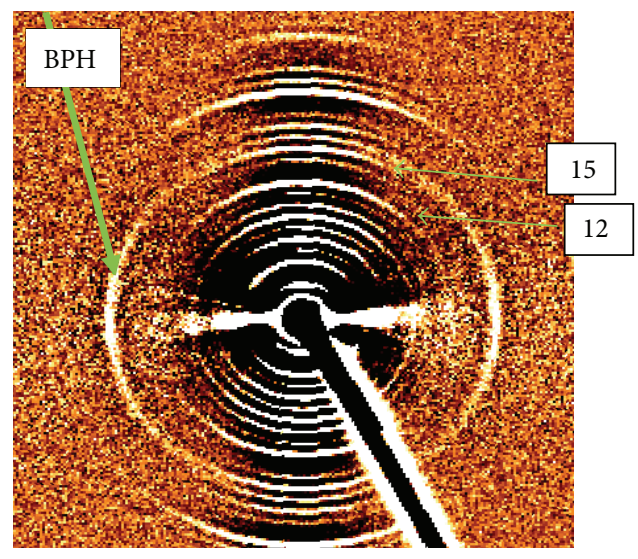

Figure 7

according to the followup FDA test. Shown in Figure 6 are high grade prostate cancer (a), low grade prostate cancer (b), and the pattern obtained from cured (c).

Another problem that some men experience is an enlarged prostate. This may not be due to cancer but may be due to benign prostate hypertrophy $(\mathrm{BPH})$. The change in the diffraction pattern for BPH was shown in Figure 3 associated with low angle prostate cancer. In Figure 7, the $\mathrm{BPH}$ ring is on its own. It is situated between the 15th and the 16th orders, indicated by the green arrow. This $\mathrm{BPH}$ ring is similar to that for breast cancer which is rare in men and can be tested by fibre diffraction of hair.

Other cancers such as melanoma and lung cancer have different rings of change, as reported elsewhere [11, 14].

\section{Conclusions}

These FDA diagnostic changes appear earlier and have a greater degree of accuracy than is currently possible with existing medical technology. Using this more accurate and sensitive FDA diagnostic test, prostate specific antigen (PSA) 
tests and painful biopsies of the prostate will not be necessary, as the FDA test would only need to be followed by an MRI (magnetic resonance imaging). This highly accurate diagnostic test is noninvasive and could be run on a yearly basis from an early age thus locating prostate cancers at a very early stage, where safe successful removal is possible. FDA would therefore greatly reduce the fatalities for this cancer. With the possibility that this skin can be done in local pathology clinics with the use of the latest rotating anodes the price would also be affordable.

\section{Ethical Approval}

Uniting Care Health Human Research Committee granted ethics approval for this study (Wesley Hospital).

\section{Conflict of Interests}

The authors declare that there is no conflict of interests regarding the publication of this paper.

\section{Acknowledgments}

The authors acknowledge the generous support of the BioCAT facility (Advanced Photon Source), Argonne National Laboratory, and the assistance provided by the beam-line staff of this facility. The Advanced Photon Source is supported by the US Department of Energy, Basic Energy Sciences and Office of Science under Contract no. W-31-109-Eng-38. BioCAT is a National Institute of Health supported Research Centre, RR-08630. They are also grateful to Professor M. McGovern for assistance with drafting this paper.

\section{References}

[1] V. J. James, “Using physics to diagnose cancer," Biophysical Reviews and Letters, 2014.

[2] B. Brodsky, E. F. Eikenberry, and K. Cassidy, "An unusual collagen periodicity in skin," Biochimica et Biophysica Acta, vol. 621, no. 1, pp. 162-166, 1980.

[3] R. H. Stinson and P. R. Sweeny, "Skin collagen has an unusual d-spacing," Biochimica et Biophysica Acta, vol. 621, no. 1, pp. 158$161,1980$.

[4] S. G. Tomlin and C. R. Worthington, "Amianthoid change: orientation of normal collagen fibrils during aging," Proceedings of the Royal Society of London A, vol. 235, no. 1201, pp. 189-201, 1956.

[5] D. W. Hukins, D. P. Knight, and J. Woodhead-Galloway, "Aminthoid change: orientation of normal collagen fibrils during aging," Science, vol. 194, no. 4265, pp. 622-624, 1976.

[6] V. J. James, L. Delbridge, S. V. McLennan, and D. K. Yue, "Use of X-ray diffraction in study of human diabetic and aging collagen," Diabetes, vol. 40, no. 3, pp. 391-394, 1991.

[7] V. J. James, "Synchrotron fibre diffraction identifies and locates foetal collagenous breast tissue associated with breast carcinoma," Journal of Synchrotron Radiation, vol. 9, no. 2, pp. 71-76, 2002.

[8] V. J. James and B. E. Willis, "Molecular changes in skin predict predisposition to breast cancer," Journal of Medical Genetics, vol. 39, no. 2, article E1, 2002.
[9] J. P. Bradshaw and A. Miller, "Osteogenesis imperfecta: an x ray fibre diffraction study," Annals of the Rheumatic Diseases, vol. 45, no. 9, pp. 750-756, 1986.

[10] V. J. James, "Fiber diffraction of skin and nails provides an accurate diagnosis of malignancies," International Journal of Cancer, vol. 125, no. 1, pp. 133-138, 2009.

[11] V. J. James and N. Kirby, "The connection between the presence of Melanoma and changes in fibre diffraction patterns," Cancers, vol. 2, no. 2, pp. 1155-1165, 2010.

[12] V. James, G. Corino, T. Robertson et al., "Early diagnosis of breast cancer by hair diffraction," International Journal of Cancer, vol. 114, no. 6, pp. 969-972, 2005.

[13] V. J. James, "Extremely early diagnostic test for prostate cancer," Journal of Cancer Therapy, vol. 2, no. 3, pp. 377-380, 2011.

[14] V. J. James, "A review of low angle fibre diffraction in the diagnosis of disease," British Journal of Medicine and Medical Research, vol. 3, no. 2, pp. 383-397, 2013.

[15] Australian Institute of Health and Welfare, ACIM (Australian Cancer Incidence and Mortality) Books, AIHW, Canberra, Australia, 2014.

[16] J. Ferlay, I. Soerjomataram, M. Ervik et al., GLOBOCAN 2012 v1.0, Cancer Incidence and Mortality Worldwide: IARC Cancer Base No. 11, International Agency for Research on Cancer, Lyon, France, 2013, http://globocan.iarc.fr/.

[17] V. James, "False-positive results in studies of changes in fiber diffraction of hair from patients with breast cancer may not be false," Journal of the National Cancer Institute, vol. 95, no. 2, pp. 170-171, 2003. 


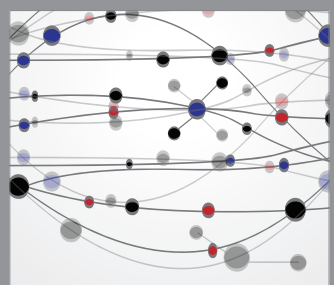

The Scientific World Journal
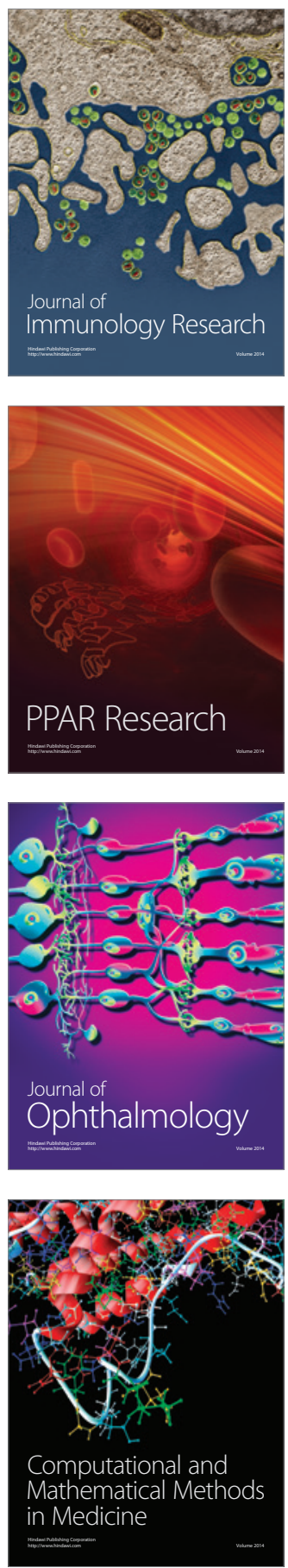

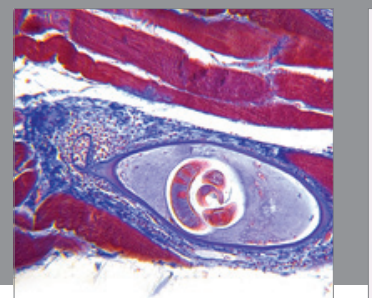

Gastroenterology

Research and Practice
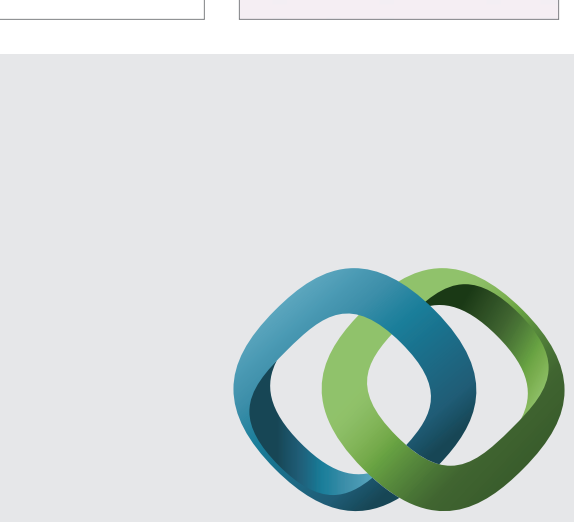

\section{Hindawi}

Submit your manuscripts at

http://www.hindawi.com
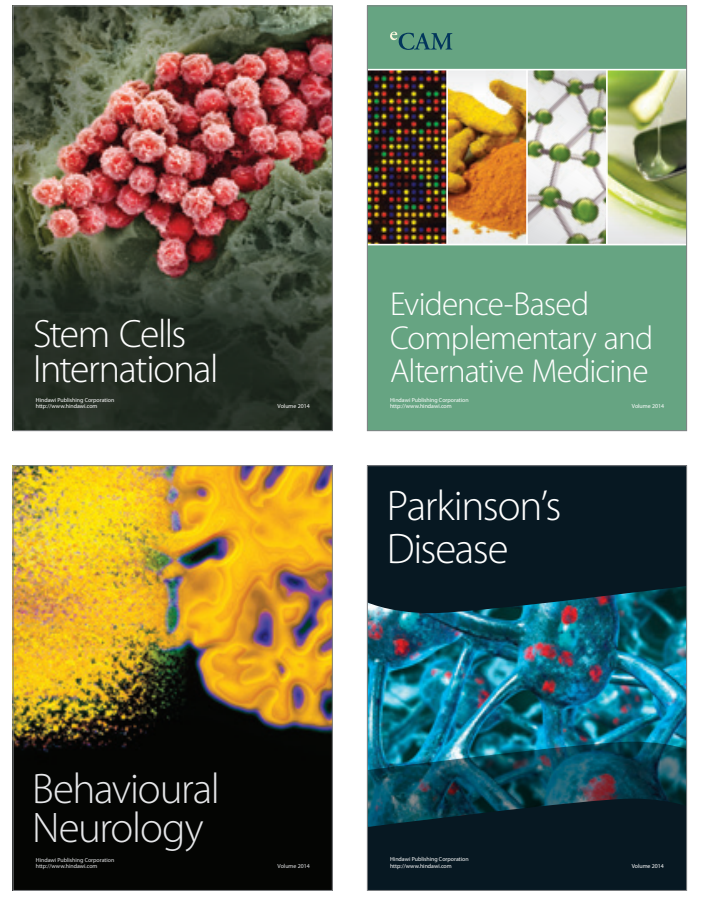
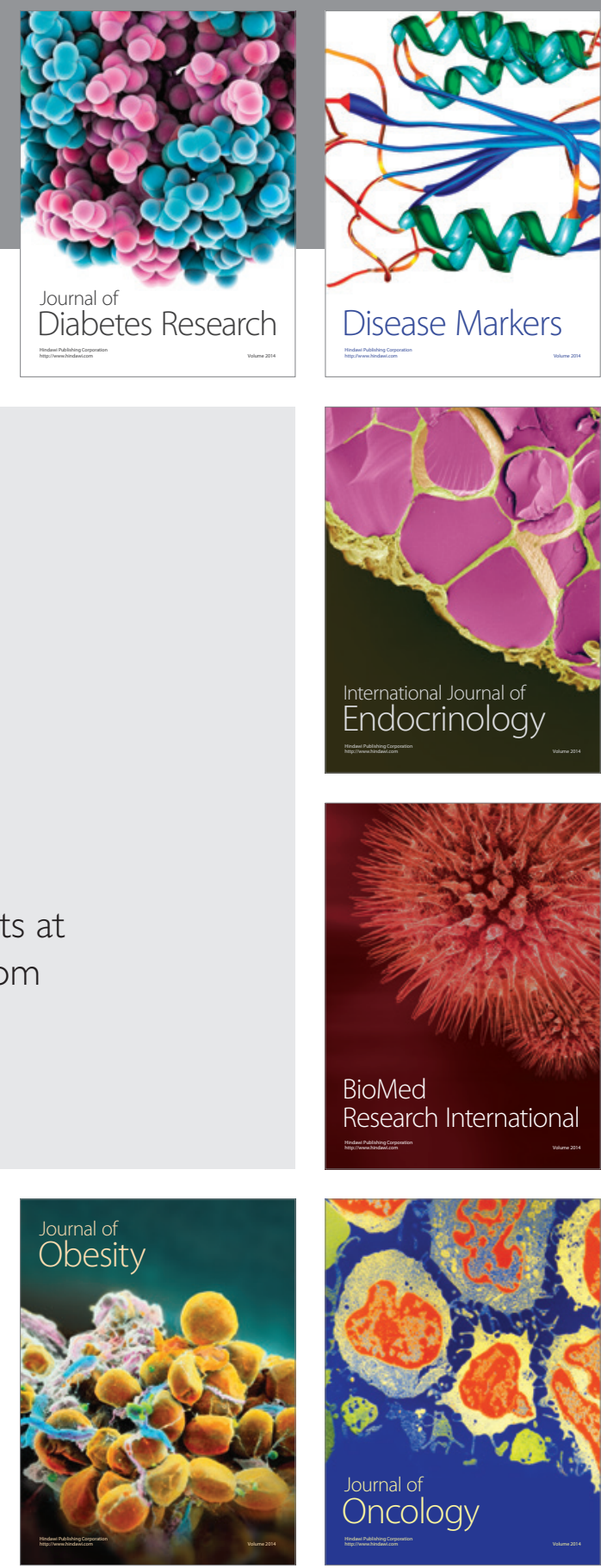

Disease Markers
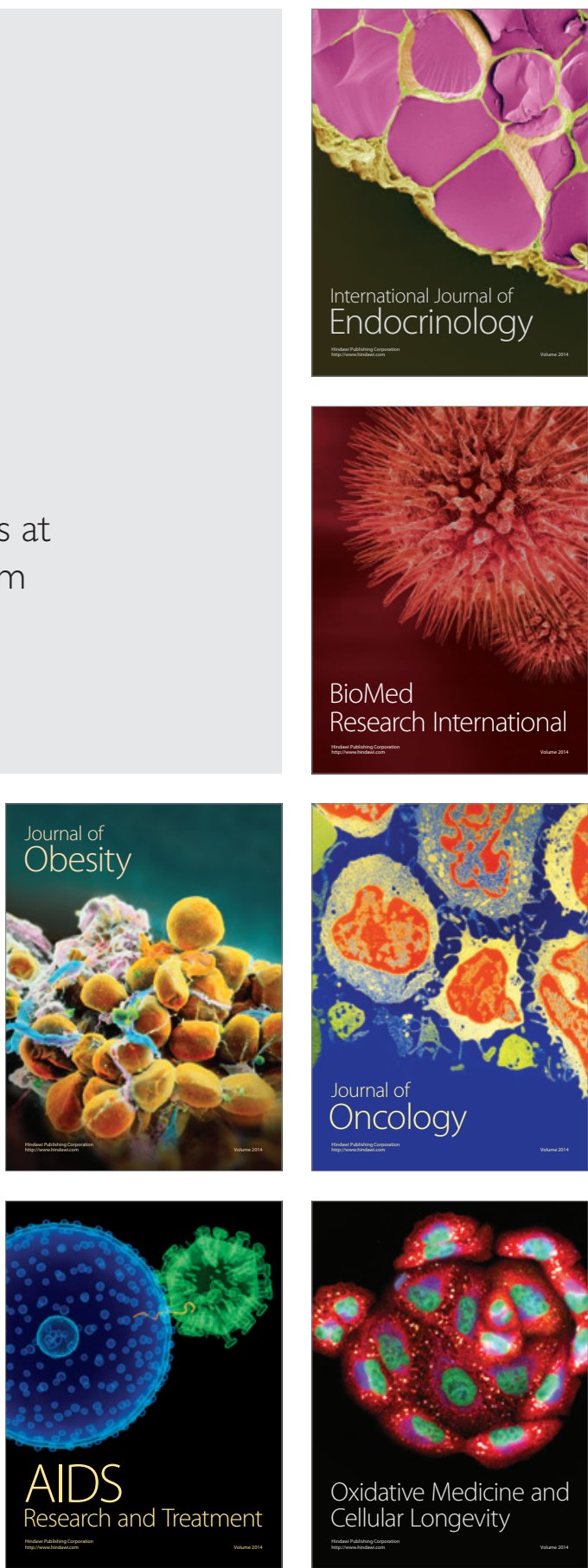